\title{
THE COALGEBRAIC ENRICHMENT OF ALGEBRAS IN HIGHER CATEGORIES
}

\author{
MAXIMILIEN PÉROUX
}

\begin{abstract}
We prove that given $C$ a presentably symmetric monoidal $\infty$-category, and any essentially small $\infty$-operad $\mathcal{O}$, the $\infty$-category of $\mathcal{O}$-algebras in $C$ is enriched, tensored and cotensored over the presentably symmetric monoidal $\infty$-category of $\mathcal{O}$-coalgebras in $\mathcal{C}$. We provide a higher categorical analogue of the universal measuring coalgebra. For categories in the usual sense, the result was proved by Hyland, López Franco, and Vasilakopoulou.
\end{abstract}

\section{INTRODUCTION}

The dual of a coalgebra is always an algebra. However, unless we require the algebra to be finite dimensional, the dual of an algebra is not a coalgebra. The universal measuring coalgebra was introduced in [Swe69] as a way to balance this issue. In ordinary categories, the measuring provides an enrichment for algebras over coalgebras: this was established in [HLV17, 5.2] and [Vas19, 2.18]. We provide here, in Theorem 3.19, its $\infty$-categorical analogue. In any presentably symmetric monoidal $\infty$-category, the algebra objects are enriched, tensored and cotensored over coalgebras. Therefore spaces of algebra morphisms are endowed with a rich structure. We use the notion of enriched $\infty$-categories following [GH15] and Hin18.

Algebras in $\infty$-categories formalize the notion of homotopy coherent associative and unital algebras, see Lur17. Following Lur18a, we provide a general dual definition of coalgebras in $\infty$-categories. These are objects with a comultiplication that is coassociative up to higher homotopies. We show, in Proposition 2.8, that if an $\infty$-operad $\mathcal{O}$ is essentially small, the $\infty$-category of $\mathcal{O}$-coalgebras in a presentable $\infty$-category remains presentable.

A similar result would be very challenging to prove in model categories. Let $\mathrm{M}$ be a combinatorial symmetric monoidal model category. Suppose we have a model structure for algebras $A \lg (M)$ in $M$ and a model structure for coalgebras $\operatorname{CoAlg}(\mathrm{M})$ in $\mathrm{M}$, in which the weak equivalences in both of these models are created by their underlying functor. One analogous result would be to show that $\operatorname{Alg}(M)$ is a CoAlg(M)-model category, in the sense of [Hov99, 4.2.18]. There are several issues with that. A left-induced model structure on $\operatorname{CoAlg}(\mathrm{M})$ may not always exist, and when it does, $\mathrm{M}$ may have been replaced by a Quillen equivalent model category that is not a monoidal model category, see [HKRS17. Even in cases where we can left-induce from a monoidal model category, the homotopy theory associated to CoAlg(M) may not be the correct one, see PS19] and Pér20a].

2010 Mathematics Subject Classification. 16T15, 18C35, 18D10, 18D20, 18N70, 55P43.

Key words and phrases. algebra, coalgebra, enrichment, operads, $\infty$-categories, presentable. 
Acknowledgement. The results here are part of my $\mathrm{PhD}$ thesis Pér20b, and as such, I would like to express my gratitude to my advisor Brooke Shipley for her help and guidance throughout the years. I would also like to thank Rune Haugseng for clarifying and answering many of my questions. I am also thankful for many fruitful conversations with Shaul Barkan and tslil clingman that sparked results in this paper. I thank the referee for helpful comments on the preliminary version of this paper.

\section{Presentability of Coalgebras}

We present here the formal definition of coalgebras in $\infty$-categories, generalizing Lur18a, Section 3.1], which was for the case of $\mathbb{E}_{\infty}$-coalgebras. We define and extend the results for coalgebras over any $\infty$-operad. Our main result in this section is that coalgebras of a presentably symmetric monoidal $\infty$-category also form a presentable $\infty$-category, see Corollary 2.9.

We invite the reader to review the definition of a symmetric monoidal $\infty$-category in [Lur17, 2.0.0.7]. More generally, for any $\infty$-operad $\mathcal{O}$ (see [Lur17, 2.1.1.10]), we will consider the notion of an $\mathcal{G}$-monoidal $\infty$-category as in [Lur17, 2.1.2.15]. If we choose $\mathcal{O}$ to be the commutative $\infty$-operad ([Lur17, 2.1.1.18]), then $\mathcal{O}$-monoidal $\infty$-categories are precisely symmetric monoidal $\infty$-categories.

Definition 2.1. Let $\mathcal{O}$ be an $\infty$-operad. Let $C$ be an $\mathcal{O}$-monoidal $\infty$-category. An $\mathcal{O}$-coalgebra object in $\mathcal{C}$ is an $\mathcal{G}$-algebra object in $C^{\text {op }}$. The $\infty$-category of $\mathcal{O}$ coalgebra objects in $C$ is defined as the $\infty$-category $\mathcal{C o} \mathcal{A} \ell g_{\mathcal{O}}(C):=\left(\mathcal{A} \ell g_{\mathcal{O}}\left(C^{\mathrm{op}}\right)\right)^{\mathrm{op}}$. More generally, given any map $\mathcal{O}^{\prime \otimes} \rightarrow \mathcal{O}^{\otimes}$ of $\infty$-operads, we define the $\infty$-category of $\mathcal{O}^{\prime}$-coalgebras in $C$ as $\operatorname{Co} \mathcal{A} g_{\mathcal{O}^{\prime} / \mathcal{O}}(C)=\left(\mathcal{A}^{\ell} g_{\mathcal{O}^{\prime} / \mathcal{O}}\left(C^{\text {op }}\right)\right)^{\text {op }}$.

Remark 2.2. If $C$ is an $\mathcal{O}$-monoidal $\infty$-category, then $C^{\text {op }}$ can be given an $\mathcal{O}$ monoidal structure uniquely up to contractible choice, as in [Lur17, 2.4.2.7]. One can use the work of [BGN18] to give an explicit choice of the coCartesian fibration for $C^{\mathrm{op}}$. For instance, let $p: \mathcal{C}^{\otimes} \rightarrow \mathcal{O}^{\otimes}$ be the coCartesian fibration associated to the symmetric monoidal structure of $C$. Then straightening of the coCartesian fibration gives a functor:

$$
F: \mathcal{O}^{\otimes} \longrightarrow \widehat{C a t}_{\infty}
$$

where $\widehat{C a t}_{\infty}$ is the $\infty$-category of (not necessarily small) $\infty$-categories, as in Lur17, 3.0.0.5]. Then, by [BGN18, 1.5], the functor $F$ also classifies a Cartesian fibration:

$$
p^{\vee}:\left(e^{\otimes}\right)^{\vee} \longrightarrow\left(\mathcal{O}^{\otimes}\right)^{\text {op }}
$$

An explicit construction is given in [BGN18, 1.7]. The opposite map:

$$
\left(p^{\vee}\right)^{\mathrm{op}}:\left(\left(C^{\otimes}\right)^{\vee}\right)^{\mathrm{op}} \longrightarrow \mathcal{O}^{\otimes}
$$

is a coCartesian fibration that is classified by:

$$
\mathcal{O}^{\otimes} \stackrel{F}{\longrightarrow} \widehat{\operatorname{Cat}}_{\infty} \stackrel{\text { op }}{\longrightarrow} \widehat{\operatorname{Cat}}_{\infty} .
$$

One can check that the fiber of $\left(p^{\vee}\right)^{\text {op }}$ over $X$ in $\mathcal{O}$ is equivalent to $\left(C_{X}\right)^{\text {op }}$, and thus gives $C^{\text {op }}$ a $\mathcal{O}$-monoidal structure. We see that $\mathcal{O}$-coalgebras are sections of the Cartesian fibration $p^{\vee}:\left(C^{\otimes}\right)^{\vee} \rightarrow\left(\mathcal{O}^{\otimes}\right)^{\text {op }}$ that sends inert morphisms in $\left(\mathcal{O}^{\otimes}\right)^{\text {op }}$ to $p^{\vee}$-Cartesian morphisms in $\left(e^{\otimes}\right)^{\vee}$. 
Remark 2.3. Recall from Lur17, 2.0.0.1] that given any symmetric monoidal (ordinary) category $\mathrm{C}$, one can define a category $\mathrm{C}^{\otimes}$, such that the nerve $\mathscr{N}\left(\mathrm{C}^{\otimes}\right)$ is a symmetric monoidal $\infty$-category whose underlying $\infty$-category is $\mathscr{N}(\mathrm{C})$, see $\operatorname{Lur} 17$, 2.1.2.21]. If we denote by $\operatorname{CoAlg}(\mathrm{C})$ the category of coassociative and counital coalgebras in C, then, dually from [Gro20, 4.21], we obtain:

$$
\operatorname{Co} \mathcal{A} \operatorname{gg}_{\mathbb{A}_{\infty}}(\mathscr{N}(\mathrm{C})) \simeq \mathscr{N}(\operatorname{CoAlg}(\mathrm{C})) .
$$

Similarly, if we denote by $\operatorname{CoCAlg}(\mathrm{C})$ the category of cocommutative coalgebras in C, we obtain:

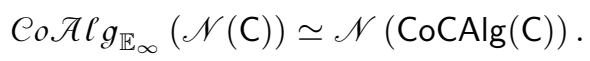

Proposition 2.4 ([Lur17, 3.2.4.4]). Let $\mathcal{G}$ be an $\infty$-operad. Let $\mathcal{C}$ be an $\mathcal{O}$ monoidal $\infty$-category. Then the $\infty$-category $\mathcal{A} \mathcal{l}_{g_{\mathcal{O}}}(\mathcal{C})$ inherits a $\mathcal{O}$-monoidal structure, given by pointwise tensor product. Dually, the $\infty$-category $\mathcal{C o}_{\mathcal{A}} g_{\mathcal{\Theta}}(C)$ inherits a $\mathcal{O}$-monoidal structure, given by pointwise tensor product.

Proposition 2.5. Let $C$ be a $\mathcal{O}$-monoidal $\infty$-category and let $K$ be a simplicial set. If, for each $X$ in $\mathcal{G}$, the fiber $C_{X}$ admits $K$-indexed colimits, then the $\infty$-category $\mathrm{Co}_{\mathcal{A l g}}(C)$ admits $K$-indexed colimits, and the forgetful functor $U$ :

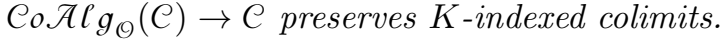

Proof. Apply [Lur17, 3.2.2.5] to the coCartesian fibration $\left(p^{\vee}\right)^{\mathrm{op}}:\left(\left(C^{\otimes}\right)^{\vee}\right)^{\mathrm{op}} \longrightarrow$ $\mathcal{O}^{\otimes}$ defined in Remark 2.2 .

Recall the definition [Lur09, 5.5.0.1] of a presentable $\infty$-category. Denote $\mathscr{P}_{r}{ }^{L}$ the $\infty$-category of presentable $\infty$-categories with small colimit preserving functors. It is endowed with a symmetric monoidal structure ([Lur17, 4.8.1.15]).

Definition 2.6. An $\infty$-category $C$ is said to be presentably $\mathcal{G}$-monoidal if it is an $\mathcal{O}$-algebra in $\mathscr{P}_{r}{ }^{L}$, i.e., $C$ is $\mathcal{O}$-monoidal, for each object $X$ in $\mathcal{O}^{\otimes}$, the fiber $C_{X}^{\otimes}$ is presentable, and for every morphism $f: X \rightarrow Y$ in $\mathcal{O}^{\otimes}$, the associated functor $f_{!}: C_{X}^{\otimes} \rightarrow C_{Y}^{\otimes}$ preserves small colimits.

Example 2.7. When $\mathcal{O}^{\otimes}$ is the commutative $\infty$-operad (see [Lur17, 2.1.1.18]), then a presentably $\mathcal{O}$-monoidal $\infty$-category is called presentably symmetric monoidal. Notice that a symmetric monoidal $\infty$-category $C$ is presentably symmetric monoidal if and only if $C$ is presentable and the tensor product $\otimes: C \times C \rightarrow C$ preserves small colimits in each variable.

The following dualizes the result on algebras in [Lur17, 3.2.3.5] and generalizes the result for cocommutative coalgebras in [Lur18a, 3.1.4].

Proposition 2.8. Let $\mathcal{O}$ be an essentially small $\infty$-operad. Let $C$ be a presentably $\mathcal{\Theta}$-monoidal $\infty$-category. Then $\operatorname{Co} \mathcal{A} g_{\mathcal{O}}(C)$ is a presentably $\mathcal{\Theta}$-monoidal $\infty$-category.

Proof. By Propositions 2.4 and 2.5. we only need to check that $\operatorname{Co} \mathcal{A} g_{\mathcal{O}}(C)$ is presentable. Denote the coCartesian fibration $p: C^{\otimes} \rightarrow \mathcal{O}^{\otimes}$ that defines the $\mathcal{O}$ monoidal structure of $C$. We apply [Lur09, 5.4.7.11, 5.4.7.14] to the subcategory $\mathscr{D}_{r}{ }^{L}$ of $\widehat{C a t}_{\infty}$, to the Cartesian fibration $p^{\vee}:\left(\mathcal{C}^{\otimes}\right)^{\vee} \rightarrow\left(\mathcal{O}^{\otimes}\right)^{\text {op }}$ described in Remark 2.2. and the set of inert morphisms in $\left(\mathcal{O}^{\otimes}\right)^{\mathrm{op}}$. The subcategory $\mathscr{D}_{\tau}{ }^{L}$ respects the conditions (a), (b) and (c) of [Lur09, 5.4.7.11] by Lur09, 5.4.3.13, 5.5.3.6, 5.1.2.4]. 
Therefore we only need to check that the fibers of $p^{\vee}$ over any object of $\left(\mathcal{C}^{\otimes}\right)^{\text {op }}$ are presentable, and that the associated functors between the fibers, induced by the Cartesian structure of $p^{\vee}$, are accessible.

For any object $X$ in $\mathcal{O}^{\otimes}$, the fiber of $p^{\vee}$ over $X$ is equivalent to the fiber $C_{X}^{\otimes}$ of $p$ over $X$. By Definition 2.6, the fibers over $p$ are presentable and the associated functors $C_{X} \rightarrow C_{Y}$ are accessible maps for any morphism $X \rightarrow Y$ in $\mathcal{C}^{\otimes}$. Thus the induced maps $\left(\left(C^{\otimes}\right)^{\vee}\right)_{Y} \rightarrow\left(\left(C^{\otimes}\right)^{\vee}\right)_{X}$ are also accessible, as $C^{\otimes}$ and $\left(C^{\otimes}\right)^{\vee}$ have the same underyling $\infty$-category $C$ by BGN18, 1.3].

Corollary 2.9. Let $\mathcal{G}$ be an essentially small $\infty$-operad. If $C$ is a presentably symmetric monoidal $\infty$-category, then $\mathrm{Co} \mathcal{A} \mathcal{l g}_{\mathcal{\Theta}}(C)$ is a presentably symmetric monoidal $\infty$-category.

Remark 2.10. In general, if $C$ is compactly generated (Lur09, 5.5.7.1]), there is no guarantee that $\operatorname{Co}_{\mathcal{A}} \mathrm{lg}_{\mathcal{O}}(C)$ is also compactly generated. However, the fundamental theorem of coalgebras (see [Swe69, II.2.2.1] or [GG99, 1.6]) states that if $C$ is (the nerve of) vector spaces, or chain complexes over a field, then $\mathcal{C} \mathcal{A} \ell g_{\mathbb{A}_{\infty}}(C)$ is compactly generated and the forgetful functor $U: \mathcal{C o}_{\mathcal{A}} \mathcal{G}_{\mathbb{A}_{\infty}}(C) \rightarrow C$ preserves and reflects compact objects. From [AP04, 4.2], if $\kappa$ is an uncountable regular cardinal, we conjecture that the fundamental theorem of coalgebra can be expended in the

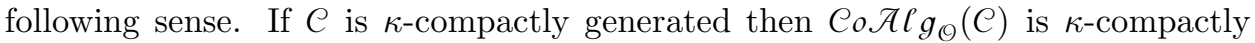
generated and the forgetful functor preserves and reflects $\kappa$-compact objects.

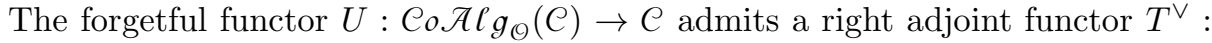

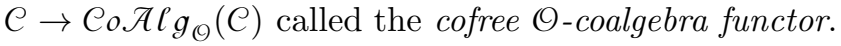

Corollary 2.11. Let $\mathcal{G}$ be an essentially small $\infty$-operad. Let $\mathcal{C}$ be a presentably $(\mathcal{C}$-monoidal $\infty$-category. Then there is forgetful-cofree adjunction:

$$
U: \operatorname{Co}^{A} \lg _{\mathcal{O}}(C) \longleftrightarrow \stackrel{\perp}{\longleftarrow} C: T^{\vee}
$$

Proof. Apply Propositions 2.5 and 2.8 and the adjoint functor theorem Lur09, $5.5 .2 .9]$.

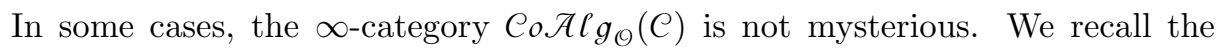
following result from Lurie. Let $C$ be a symmetric monoidal $\infty$-category, and denote by $C_{\mathrm{fd}}$ the full subcategory spanned by the dualizable objects, see [Lur17, 4.6.1]. It inherits a symmetric monoidal structure. For each dualizable object $X$, we denote $X^{\vee}$ its dual and this defines a contravariant endofunctor on $C_{\mathrm{fd}}$.

Proposition 2.12 ([Lur18a, 3.2.4]). Let C be a symmetric monoidal $\infty$-category. Then taking dual objects assigns an equivalence of symmetric monoidal $\infty$-categories $\left(C_{\mathrm{fd}}\right)^{\text {op }} \stackrel{\simeq}{\longrightarrow} C_{\mathrm{fd}}$. In particular, for any $\infty$-operad $\mathcal{O}$, we obtain an equivalence

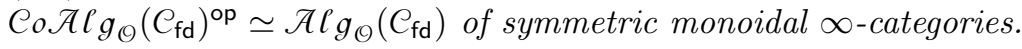

The anti-equivalence above has been generalized to a wider class in [BP20, 3.31].

\section{The Universal Measuring Coalgebra}

Classically, in any presentable symmetric monoidal closed ordinary category, the category of monoids is enriched, tensored and cotensored in the symmetric monoidal category of comonoids. This was proven in [HLV17, 5.2] and [Vas19, 2.18]. See also the example of the differential graded case in [AJ13. We show here in Theorem 3.19 an equivalent statement in $\infty$-categories. 
An $\infty$-category shall be defined to be enriched over a symmetric monoidal $\infty$ category in the sense of GH15]. Alternatively, the reader can use the definition in Hin18, 3.1.2, 7.1.1(2)]. By [Hin18, 3.4.4] they are equivalent. The author in Hin18] uses the term precategory instead of enriched category for various reasons that are explained in [Hin18, 4.8], but the reader can safely ignore those technicalities and think of them as enriched $\infty$-categories for all the results we quote in this paper.

An $\infty$-category is tensored or cotensored over a monoidal $\infty$-category in the sense of [Lur17, 4.2.1.19] or [Lur17, 4.2.1.28] respectively. Our desired enrichment in Theorem 3.19 will also be enriched in the sense of [Lur17, 4.2.1.28]. It is shown in Hei20, that the definitions of enrichment of Lurie and Gepner-Haugseng are equivalent.

Throughout this section, let $C$ be a presentably symmetric monoidal $\infty$-category. It is in particular closed, and thus the strong symmetric monoidal functor:

$$
\otimes: C \times C \longrightarrow C
$$

induces a lax symmetric monoidal functor $[-,-]: C^{\text {op }} \times C \rightarrow C$, see [Hau20, I.3], characterized by the universal mapping property $\mathcal{C}(X \otimes Y, Z) \simeq \mathcal{C}(X,[Y, Z])$, for all $X, Y$, and $Z$ in $C$. In other words, the functor $-\otimes Y: C \rightarrow C$ is a left adjoint to $[Y,-]: C \rightarrow C$.

3.1. The Sweedler cotensor. Let $\mathcal{O}$ be an essentially small $\infty$-operad. From the lax symmetric monoidal structure of $[-,-]: C^{\mathrm{op}} \times C \rightarrow C$, we obtain a functor:

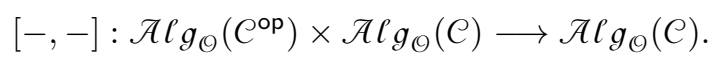

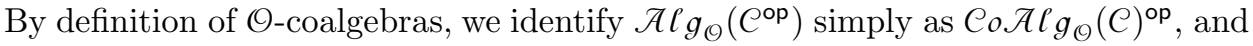
thus obtain the following definition.

Definition 3.1. Let $C$ and $\mathcal{O}$ be as above. We call the induced functor:

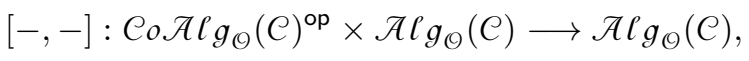

the Sweedler cotensor. In the literature, it is sometimes called the convolution algebra or the convolution product, see [Swe69, 4.0] and [AJ13].

Remark 3.2. The term convolution product stems from the algebra structure that generalizes the usual convolution product in representation theory. See HGK10, 2.12.3]. It also generalizes the classical convolutions of real functions of compact support, see [HGK10, 2.14.4].

Example 3.3. The Sweedler cotensor in the case where $\mathcal{O}=\mathbb{E}_{\infty}$ and $C$ is the $\infty$-category of $R$-modules in a symmetric monoidal $\infty$-category, where $R$ is an $\mathbb{E}_{\infty}$-algebra, was presented in [Lur18b, Section 1.3.1]. See also [Nik16, 6.6].

Example 3.4. Let $\mathbb{I}$ be the unit of the symmetric monoidal structure of $C$. Let $C$ be any $\mathcal{O}$-coalgebra. The Sweedler cotensor $[C, \mathbb{I}]$ is the linear dual $C^{*}$. Therefore the linear dual of an $\mathcal{O}$-coalgebra is always an $\mathcal{O}$-algebra. In particular the linear dual functor $(-)^{*}: C^{\text {op }} \rightarrow C$ lifts to the Sweedler cotensor $(-)^{*}=[-, \mathbb{I}]$ : $\operatorname{Co} \mathcal{A} g_{\mathcal{\Theta}}(C)^{\text {op }} \rightarrow \mathcal{A} \lg _{\mathcal{\Theta}}(C)$. Here we recover the classical result that the dual of a coalgebra is always an algebra, see [Swe69, 1.1.1]. More precisely, if $C$ is a coalgebra with comultiplication $\Delta: C \rightarrow C \otimes C$ and counit $\varepsilon: C \rightarrow \mathbb{I}$, then $C^{*}$ is a coalgebra with multiplication:

$$
C^{*} \otimes C^{*} \longrightarrow(C \otimes C)^{*} \stackrel{\Delta^{*}}{\longrightarrow} C^{*}
$$


where the unlabeled map is given by the lax monoidal structure of the linear dual. The unit is given by using the equivalence $\mathbb{I}^{*} \simeq \mathbb{I}$.

Remark 3.5. In a presentably symmetric monoidal $\infty$-category $C$, given an object $X$ that is dualizable (see Lur17, 4.6.1]), the dual of $X$ is given precisely by its linear dual $X^{*}$ (see BP20, 3.10]). Thus, the above defined functor $(-)^{*}$ :

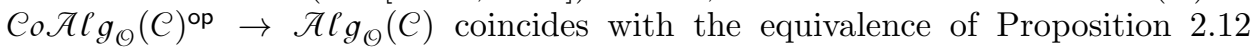
$(-)^{\vee}: \operatorname{Co}^{A} \ell_{g_{\Theta}}\left(C_{\mathrm{fd}}\right)^{\mathrm{op}} \stackrel{\simeq}{\longrightarrow} \mathcal{A} g_{g_{\mathcal{\Theta}}}\left(C_{\mathrm{fd}}\right)$, when we restrict $(-)^{*}$ to the subcategory $\operatorname{Co} \mathcal{A} g_{g_{\Theta}}\left(C_{\mathrm{fd}}\right)^{\mathrm{oP}}$.

3.2. The Sweedler tensor. Since $[-,-]: C^{\mathrm{op}} \times C \rightarrow C$ is a continuous functor in both variables, and limits in $\mathcal{A} \ell g_{\odot}(C)$ are computed in $C$, we get that the Sweedler cotensor is a continuous functor in both variables. Fix $C$ an $\mathcal{G}$-coalgebra in $C$. Then the continuous functor:

$$
[C,-]: \mathcal{A} \lg _{\mathfrak{\Theta}}(C) \rightarrow \mathcal{A} \lg _{\Theta}(C),
$$

is accessible (as filtered colimits in $\mathcal{A} \ell g_{\Theta}(C)$ are computed in $C$ ) and is between presentable $\infty$-categories. Therefore, by the adjoint functor theorem Lur09, 5.5.2.9], the functor $[C,-]$ admits a left adjoint denoted $C \triangleright-: \mathcal{A} \ell g_{\mathcal{O}}(C) \rightarrow \mathcal{A} \ell g_{\mathcal{O}}(C)$.

Definition 3.6. Let $C$ and $\mathcal{O}$ be as above. We call the induced functor:

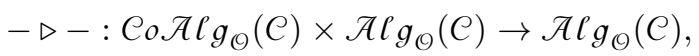

the Sweedler tensor. Previously, it was called the Sweedler product in AJ13] and later in Vas19. For $C$ a fixed $\mathcal{O}$-coalgebra, the functor $C \triangleright-$ is left adjoint to $[C,-]$ and we have in particular the equivalence of spaces:

$$
\mathcal{A} \lg _{\mathcal{\Theta}}(C \triangleright A, B) \simeq \mathcal{A} \ell_{g_{\Theta}}(A,[C, B]),
$$

for any $\mathcal{O}$-algebras $A$ and $B$.

Example 3.7. In AJ13, 3.4.1], an explicit formula of the Sweedler tensor was given in the discrete differential graded case.

3.3. The Sweedler hom. Let now $A$ be an $\mathcal{O}$-algebra in $\mathcal{C}$. The continuous functor:

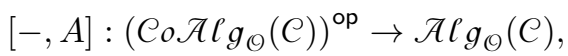

induces a cocontinuous functor on its opposites:

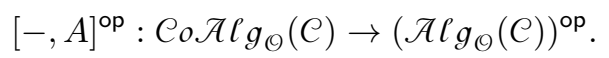

The cocontinuous functor is from a presentable $\infty$-category to an essentially locally small $\infty$-category: as the opposite of an essentially locally small $\infty$-category is also essentially locally small, and presentable $\infty$-categories are always essentially locally small. Thus, by the adjoint functor theorem [Lur09, 5.5.2.9, 5.5.2.10], the functor

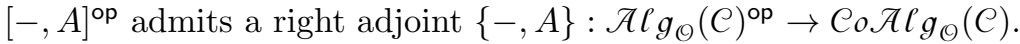

Definition 3.8. Let $C$ and $\mathcal{O}$ be as above. We call the induced functor:

$$
\{-,-\}: \mathcal{A} l g_{\mathcal{O}}(C)^{\mathrm{op}} \times \mathcal{A} l g_{\mathcal{\Theta}}(C) \rightarrow \operatorname{Co}^{A} \mathcal{l} g_{\mathcal{\Theta}}(C),
$$

the Sweedler hom. For $A$ and $B$ any $\mathcal{O}$-algebra in $\mathcal{C}$, the $\mathcal{O}$-coalgebra $\{A, B\}$ is called the universal measuring coalgebra in $C$ of $A$ and B. See [Swe69, 7.0] for the discrete case in vector spaces. In particular, if we fix $A$, we obtain that $\{-, A\}$ is the right adjoint of $[-, A]^{\mathrm{op}}$ and we have the equivalence of spaces:

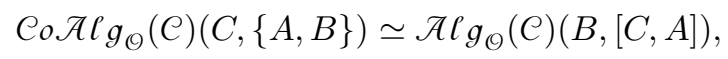


for any $\mathcal{O}$-coalgebra $C$.

Example 3.9. Let $\mathbb{I}$ be the unit of the symmetric monoidal structure of $C$. Then, for any $\mathcal{O}$-algebra $A$ in $\mathcal{C}$, define $A^{\circ}$ to be the measuring coalgebra $\{A, \mathbb{I}\}$. It is called the Sweedler dual or finite dual of the $\mathcal{O}$-algebra $A$ in $C$. In particular, we obtain a functor $(-)^{\circ}=\{-, \mathbb{I}\}^{\mathrm{op}}: \mathcal{A} \ell g_{\mathcal{\Theta}}(C) \rightarrow \mathcal{C o} \mathcal{A} \ell g_{\mathcal{O}}(C)^{\mathrm{op}}$, which is the left adjoint of the linear dual functor $(-)^{*}: \operatorname{Co}^{A} \mathcal{l} g_{\mathcal{O}}(C)^{\text {op }} \rightarrow \mathcal{A} \mathcal{l} g_{\mathcal{\Theta}}(C)$ defined in Example 3.4. In particular, we have the equivalence of spaces:

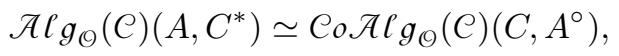

for any $\mathcal{O}$-coalgebra $C$ and any $\mathcal{G}$-algebra $A$. This was proven in the discrete classical case of vector spaces in [Swe69, 6.0.5]. By Remark 3.5, when the $\mathcal{O}$-algebra $A$ is dualizable in $C$, then $A^{\circ} \simeq A^{*}$ as an object in $C$.

Recall we have defined the cofree $\mathcal{O}$-coalgebra functor $T^{\vee}: C \rightarrow \mathcal{C o}_{\mathcal{A}} \mathcal{C} g_{\mathcal{O}}(C)$ in Corollary 2.11. We show that the Sweedler dual defined above of the free $\mathcal{O}$-algebra functor $T: C \rightarrow \mathcal{A} \ell_{g_{\mathcal{O}}}(C)$ provides an explicit description of $T^{\vee}$.

Proposition 3.10. Let $C$ be a presentably symmetric monoidal $\infty$-category. Let $\mathcal{O}$ be an essentially small $\infty$-operad. Let $X$ be an object in $\mathcal{C}$. Then the cofree $\mathcal{O}$-coalgebra on the double linear dual $X^{* *}=\left(X^{*}\right)^{*}$ is given by:

$$
T^{\vee}\left(X^{* *}\right) \simeq\left(T\left(X^{*}\right)\right)^{\circ} .
$$

Proof. Let $C$ be an $\mathcal{O}$-coalgebra in $C$. By Example 3.4 we have $U\left(C^{*}\right) \simeq U(C)^{*}$ where $U$ represents the forgetful functor on algebras or coalgebras. Then, we get the following equivalences:

$$
\begin{aligned}
\operatorname{Coflg}_{\mathcal{O}}(C)\left(C,\left(T\left(X^{*}\right)^{\circ}\right)\right) & \simeq \mathcal{A} \lg _{\mathcal{\Theta}}(C)\left(T\left(X^{*}\right), C^{*}\right) \\
& \simeq C\left(X^{*}, U(C)^{*}\right) \\
& \simeq C\left(X^{*} \otimes U(C), \mathbb{I}\right) \\
& \simeq C\left(U(C), X^{* *}\right) .
\end{aligned}
$$

Thus we obtain the desired equivalence $T^{\vee}\left(X^{* *}\right) \simeq\left(T\left(X^{*}\right)\right)^{\circ}$ by uniqueness of the right adjoint functor.

The fundamental theorem of coalgebras, as seen in Remark 2.10 provides an explicit formula for the cofree $\mathcal{O}$-coalgebra that generalizes the approach of Swe69, 6.4.1] and [GG99, 1.10].

Corollary 3.11. Let $C$ be a presentably symmetric monoidal $\infty$-category. Let $\mathcal{O}$ be an essentially small $\infty$-operad. Suppose $\mathcal{C}$ is compactly generated such that $\mathrm{Co}^{\mathcal{A} \mathrm{g}_{\mathcal{\Theta}}}(\mathcal{C})$ is also compactly generated. Suppose furthermore that every compact object $Y$ in $C$ is naturally equivalent to its double linear dual $Y \simeq Y^{* *}$. Then, for any object $X$ in $\mathcal{C}$, its cofree $\mathcal{O}$-coalgebra is given by:

$$
T^{\vee}(X) \simeq \operatorname{colim}_{i}\left(\left(T\left(X_{i}^{*}\right)\right)^{\circ}\right),
$$

where $X \simeq \operatorname{colim}_{i} X_{i}$ is a filtered colimit of compact objects $X_{i}$ in $C$.

Proof. Let $C$ be an $\mathcal{O}$-coalgebra in $C$. Since $\operatorname{Co} \mathcal{A} g_{g_{\mathcal{O}}}(C)$ is compactly generated, then $C$ is the filtered colimit of compact coalgebras $C_{j}$ :

$$
C \simeq \operatorname{colim}_{j} C_{j} .
$$


By compactness, we obtain the equivalence:

$$
\operatorname{Coflg}_{\Theta}(C)\left(C, \operatorname{colim}_{i}\left(\left(T\left(X_{i}^{*}\right)\right)^{\circ}\right)\right) \simeq \lim _{j} \operatorname{colim}_{i} \operatorname{Co}_{\mathcal{A}} g_{\mathcal{\Theta}}(C)\left(C_{j}, T\left(X_{i}^{*}\right)^{\circ}\right) \text {. }
$$

By Proposition 3.10, we obtain an equivalence:

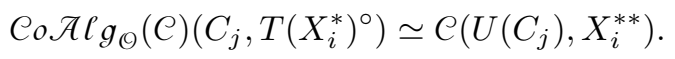

By hypothesis, the natural equivalence $X \simeq X_{i}^{* *}$ provides the equivalence:

$$
C\left(U\left(C_{j}\right), X_{i}^{* *}\right) \simeq C\left(U\left(C_{j}\right), X_{i}\right)
$$

Since $U: C o \mathcal{A} l g_{\mathcal{O}}(C) \rightarrow C$ preserves colimits and compact objects (by Proposition 2.5 and Lur09, 5.5.7.2]), we obtain that $U(C)$ is the filtered colimit of compact objects $U\left(C_{j}\right)$ in $C$. Therefore:

$$
\lim _{j} \operatorname{colim}_{i} C\left(U\left(C_{j}\right), X_{i}\right) \simeq C(U(C), X) .
$$

Thus we have shown $\mathcal{C o}_{\mathcal{A} \ell g_{\mathcal{O}}}(C)\left(C, \operatorname{colim}_{i}\left(\left(T\left(X_{i}^{*}\right)\right)^{\circ}\right)\right) \simeq \mathcal{C}(U(C), X)$. By uniqueness of the right adjoint, we obtain the desired equivalences.

We shall explain where the term universal measuring is coming from. Recall that the internal hom property of $C$ implies that, for any $X, Y$ and $Z$ objects in $C$, there is an equivalence of spaces: $\mathcal{C}(X \otimes Y, Z) \simeq \mathcal{C}(Y,[X, Z])$. The Sweedler cotensor gives conditions for an $\mathcal{O}$-algebra structure on $[X, Z]$. The following is a generalization of [Swe69, 7.0.1] and [AJ13, 3.3.1].

Definition 3.12. Let $C$ and $\mathcal{O}$ be as above. Let $C$ be an $\mathcal{O}$-coalgebra in $C$, and $A$ and $B$ be $\mathcal{O}$-algebras in $C$. Let $\psi: C \otimes A \rightarrow B$ be a map in $C$. We say that $(C, \psi)$ measures $A$ to $B$ (or $(C, \psi)$ is a measuring of $A$ to $B$ ) if the adjoint map $A \rightarrow[C, B]$ is a map of $\mathcal{O}$-algebras in $C$.

We give examples generalized from [AJ13].

Example 3.13 ([AJ13, 3.3.3]). If $\mathbb{I}$ is the unit of the symmetric monoidal structure of $C$, then a map $\mathbb{I} \otimes A \rightarrow B$ in $C$ is a measuring of $A$ to $B$ if and only if it is a map in $\mathcal{A} \lg _{\Theta}(C)$.

Example 3.14 ( AJ13, 3.3.4]). The adjoint of the identity map on $[C, A]$ is a map $C \otimes[C, A] \rightarrow A$ and is always a measuring. In particular, the evaluation $C \otimes C^{*} \rightarrow \mathbb{I}$ is always a measuring of $C^{*}$ to $\mathbb{I}$. Similarly $A^{\circ} \otimes A \rightarrow \mathbb{I}$ is a measuring of $A$ to $\mathbb{I}$. It is claimed to be the origin of the term measure in [HGK10, 2.12.10].

By definition of the Sweedler hom, as we have:

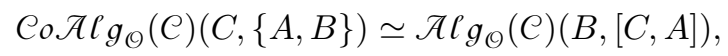

we see that the $\mathcal{O}$-coalgebra $\{A, B\}$, together with the natural map $\{A, B\} \otimes A \rightarrow B$ (adjoint of the identity over $\{A, B\}$ ), is indeed the universal measuring algebra of $A$ to $B$, in the following sense. Given any other measuring $(C, \psi)$ of $A$ to $B$, there exists a unique (up to contractible choice) map $C \rightarrow\{A, B\}$ of $\mathcal{O}$-coalgebras in $C$ such that the following diagram commutes in $C$ :

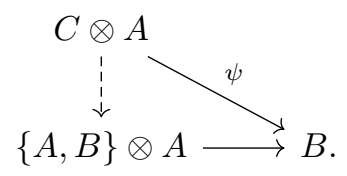


Remark 3.15. Following [AJ13, 3.3.6], we see that, given maps $A^{\prime} \rightarrow A$ and $B \rightarrow$ $B^{\prime}$ in $\mathcal{A} \lg _{\mathcal{\Theta}}(C)$, a map $C^{\prime} \rightarrow C$ in $\operatorname{Co}^{A} \ell g_{\Theta}(C)$, together with a map $A \rightarrow[C, B]$ in $\mathcal{A} \lg _{\mathcal{\Theta}}(C)$, we obtain the following map in $\mathcal{A} \ell g_{\mathcal{O}}(C)$ :

$$
A^{\prime} \longrightarrow A \longrightarrow[C, B] \longrightarrow\left[C^{\prime}, B^{\prime}\right] .
$$

This shows that the space of measurings provides a functor:

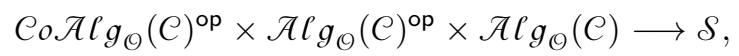

that is representable in each variable with respect to the Sweedler hom, tensor and cotensor.

Remark 3.16. We can generalize a result from BK19. Let $C$ be a presentably symmetric monoidal $\infty$-category. Let $A$ and $B$ be $\mathbb{E}_{\infty}$-algebras in $C$. Recall that the topological Hochschild homology $\operatorname{THH}(A)$ is given by tensoring over the circle:

$$
\operatorname{THH}(A) \simeq A \otimes S^{1} .
$$

In particular, if $(C, \psi)$ is a measuring of $A$ and $B$, then from the map of $\mathbb{E}_{\infty}$-algebras $A \rightarrow[C, B]$ we obtain a map of $\mathbb{E}_{\infty}$-algebras:

$$
A \otimes S^{1} \longrightarrow[C, B] \otimes S^{1} \longrightarrow\left[C, B \otimes S^{1}\right] .
$$

Therefore $(C, \psi)$ also determines a natural measuring of $\operatorname{THH}(A)$ to $\operatorname{THH}(B)$. Therefore we obtain a map of $\mathbb{E}_{\infty}$-coalgebras:

$$
\{A, B\} \longrightarrow\{\mathrm{THH}(A), \mathrm{THH}(B)\} .
$$

Remark 3.17. From AJ13, 1.3.73], the primitive elements of the measuring coalgebra $\{A, B\}$ are the derivations from $A$ to $B$. In particular, the subcoalgebra of primitive elements of the coalgebra $\{A, A\}$ is equivalent to the tangent complex of $A$.

3.4. The enrichment in coalgebras. Let $\mathscr{D}^{\otimes}$ be a monoidal $\infty$-category. Its reverse, denoted $\left(\mathscr{D}^{\otimes}\right)^{\text {rev }}$ or simply $\mathscr{D}^{\text {rev }}$, is defined in Hin18, 2.13.1]. Essentially, $\mathscr{D}$ and $\mathscr{D}^{\text {rev }}$ have the same underlying $\infty$-category but the tensor $X \otimes Y$ in $\mathscr{D}^{\text {rev }}$ corresponds precisely to $Y \otimes X$ in $\mathscr{D}$. Left modules over $\mathscr{D}$ correspond to right modules over $\mathscr{D}^{\text {rev }}$. If $\mathscr{D}$ is symmetric, then $\mathscr{D}^{\text {rev }} \simeq \mathscr{D}$ by [Hin18, 2.13.4]. We shall be interested with the reverse opposite, denoted $\mathscr{D}^{\text {rop }}=\left(\mathscr{D}^{\text {op }}\right)^{\text {rev }}$, of a monoidal $\infty$ category $\mathscr{D}$. The following is a generalization of the discrete ordinary case [HLV17, $5.1]$.

Lemma 3.18. Let $C$ and $\mathcal{O}$ be as above. Then the Sweedler cotensor endows the $\infty$-category $\mathcal{A l}_{g_{\mathcal{O}}}(\mathcal{C})$ with the structure of a right module over the reverse opposite

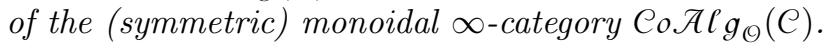

Proof. Since the internal hom $[-,-]: C \times e^{\text {op }} \rightarrow C$ is a lax symmetric monoidal functor (see [Hau20, I.3]), then it is a map of commutative algebras in $\widehat{C a t}_{\infty}$, the $\infty$-category of $\infty$-categories endowed with its Cartesian monoidal structure. This shows that $C$ is a $C^{\mathrm{op}} \times C$-algebra in $\widehat{C a t}_{\infty}$, and thus in particular, $C$ is a left module over $C^{\text {op }}$. Hence $C$ is a right module over its reverse opposite $C^{\text {rop }}$ via its internal hom. Therefore, by Proposition 2.4 the $\infty$-category $\mathcal{A} \ell g_{\mathcal{O}}(C)$ is a right module

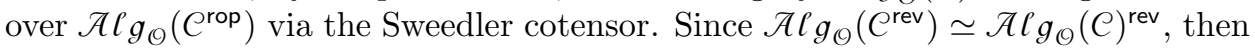

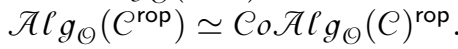


Since $\operatorname{Co} \mathcal{A l g}_{\mathcal{O}}(C)$ is a presentably symmetric monoidal $\infty$-category, it is enriched

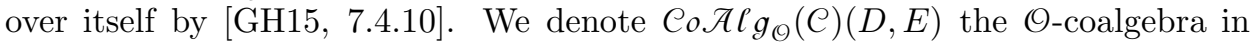

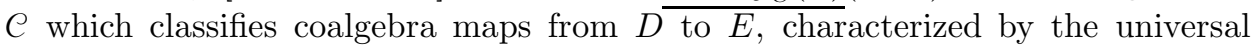
mapping property:

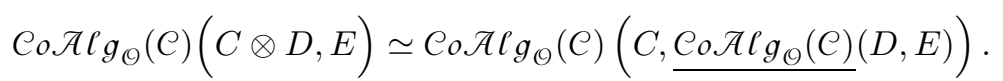

Theorem 3.19. Let $C$ be a presentably symmetric monoidal $\infty$-category. Let $\mathcal{O}$ be an essentially small $\infty$-operad. The $\infty$-category of $\mathcal{O}$-algebras $\mathcal{A}_{\mathcal{C g}_{\mathcal{O}}}(\mathcal{C})$ is enriched over the symmetric monoidal $\infty$-category $\operatorname{Co}_{\mathcal{A} \ell g_{\Theta}}(C)$, via the Sweedler hom. Moreover it is tensored and cotensored respectively using the Sweedler tensor and Sweedler cotensor. In particular, we have an equivalence of $\mathcal{O}_{\text {-coalgebras: }}$

$$
\underline{\operatorname{CoAlg}_{\mathcal{O}}(C)}(C,\{A, B\}) \simeq\{A,[C, B]\} \simeq\{C \triangleright A, B\}
$$

for any $\mathcal{O}$-coalgebra $C$ in $C$ and any $\mathcal{O}$-algebras $A$ and $B$ in $C$.

Proof. By Lemma 3.18, the $\infty$-category $\mathcal{A} \ell g_{\mathcal{O}}(C)^{\text {op }}$ is a left module over the symmetric monoidal $\infty$-category $\operatorname{Co}_{\mathcal{A}} \ell g_{\mathcal{O}}(\mathcal{C})$, via $[-,-]^{\text {op }}$ the opposite of the Sweedler

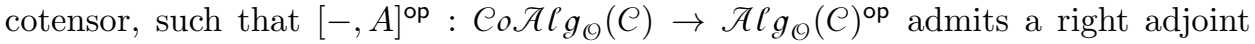
$\{-, A\}$ for all $A$ in $\mathcal{A} \ell g_{\mathcal{O}}(C)$. By [GH15, 7.4.9] (see also [Lur17, 4.2.1.33] and Hin18, 6.3.1, 7.2.1]), this shows that $\mathcal{A} \ell g_{\mathcal{O}}(C)^{\text {op }}$ is enriched over $\operatorname{Co} \mathcal{A} \ell g_{\mathcal{O}}(C)$, with tensor $[-,-]^{\mathrm{op}}$. Thus, by [Hin18, 6.2.1], we get that $\mathcal{A} \ell g_{\mathcal{O}}(C)$ is enriched over $\operatorname{CoAlg}_{\mathcal{O}}(C)$, with cotensor $[-,-]$.

Remark 3.20. The previous theorem shows that we can enrich the equivalence in Example 3.9 to an equivalence of $\mathcal{O}$-coalgebras in $C$ :

$$
\underline{\operatorname{Coflg}_{\mathcal{O}}(C)}\left(C, A^{\circ}\right) \simeq\left\{A, C^{*}\right\} \simeq(C \triangleright A)^{\circ}
$$

for any $\mathcal{O}$-coalgebra $C$ and any $\mathcal{(})$-algebra $A$.

Corollary 3.21. Let $C$ be a presentably symmetric monoidal $\infty$-category. Let $\mathcal{G}$ be an essentially small $\infty$-category. Let $A$ be an $\mathcal{G}$-algebra in $C$. Let $C$ be an $\mathcal{G}$-coalgebra in $\mathcal{C}$. Then there are adjunctions of enriched $\infty$-categories over $\operatorname{CoAlg}_{\Theta}(C)$ :

$$
\begin{aligned}
& C \triangleright-: \mathcal{A} \lg _{\mathcal{O}}(C) \longleftrightarrow \mathcal{A} \mathcal{A l g}_{\mathcal{O}}(C):[C,-] .
\end{aligned}
$$

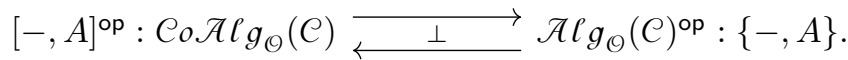

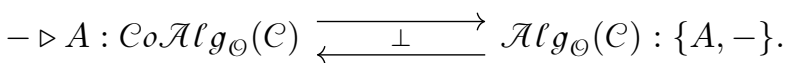

In [AJ13, 5.1.2, 5.1.4], the adjunction (3.1) generalizes adjunctions from Weil restrictions and on the de Rham algebra. The second adjunction (3.2) generalizes the anti-equivalence between finite dimensional algebras and finite dimensional coalgebras of Proposition 2.12, see also Remark 3.5] and Example 3.9 above. Finally, the adjunction (3.3) generalizes the algebraic bar-cobar adjunction as seen in AJ13. $5.3 .14]$. 


\section{REFERENCES}

[AJ13] Matthieu Anel and André Joyal, Sweedler Theory for (co)algebras and the bar-cobar constructions, 2013, arXiv:1309.6952.

[AP04] J. Adámek and H.-E. Porst, On tree coalgebras and coalgebra presentations, Theoret. Comput. Sci. 311 (2004), no. 1-3, 257-283. MR 2030299

[BGN18] Clark Barwick, Saul Glasman, and Denis Nardin, Dualizing cartesian and cocartesian fibrations, Theory Appl. Categ. 33 (2018), Paper No. 4, 67-94. MR 3746613

[BK19] Abhishek Banerjee and Surjeet Kour, On measurings of algebras over operads and homology theories, arXiv e-prints (2019), arXiv:1909.13835.

[BP20] Özgür Haldun Bayındır and Maximilien Péroux, Spanier-Whitehead duality for topological coHochschild homology, arXiv e-prints (2020), arXiv:2012.03966.

[GG99] E. Getlzer and P. Goerss, A model category structure for differential graded coalgebras, 1999, Unpublished.

[GH15] David Gepner and Rune Haugseng, Enriched $\infty$-categories via non-symmetric $\infty$ operads, Adv. Math. 279 (2015), 575-716. MR 3345192

[Gro20] Moritz Groth, A short course on $\infty$-categories, Handbook of homotopy theory (Haynes Miller, ed.), CRC Press, Boca Raton, FL, 2020, pp. 549-618.

[Hau20] Rune Haugseng, A fibrational mate correspondence for $\infty$-categories, arXiv e-prints (2020), arXiv:2011.08808.

[Hei20] Hadrian Heine, An equivalence between enriched $\infty$-categories and $\infty$-categories with weak action, arXiv e-prints (2020), arXiv:2009.02428.

[HGK10] Michiel Hazewinkel, Nadiya Gubareni, and V. V. Kirichenko, Algebras, rings and modules, Mathematical Surveys and Monographs, vol. 168, American Mathematical Society, Providence, RI, 2010, Lie algebras and Hopf algebras. MR 2724822

[Hin18] V. Hinich, Yoneda lemma for enriched infinity categories, arXiv e-prints (2018), arXiv:1309.6952, to appear in Advances in Math.

[HKRS17] Kathryn Hess, Magdalena Kędziorek, Emily Riehl, and Brooke Shipley, A necessary and sufficient condition for induced model structures, J. Topol. 10 (2017), no. 2, 324369. MR 3653314

[HLV17] Martin Hyland, Ignacio López Franco, and Christina Vasilakopoulou, Hopf measuring comonoids and enrichment, Proc. Lond. Math. Soc. (3) 115 (2017), no. 5, 1118-1148. MR 3733560

[Hov99] Mark Hovey, Model categories, Mathematical Surveys and Monographs, vol. 63, American Mathematical Society, Providence, RI, 1999. MR 1650134

[Lur09] Jacob Lurie, Higher topos theory, Annals of Mathematics Studies, vol. 170, Princeton University Press, Princeton, NJ, 2009. MR 2522659

[Lur17] Jacob Lurie, Higher algebra, https://www.math.ias.edu/ lurie/papers/HA.pdf, 2017, electronic book.

[Lur18a] _ Elliptic cohomology I, https://www.math.ias.edu/ lurie/papers/Elliptic-I.pdf 2018, unpublished.

[Lur18b] _ Elliptic cohomology II: Orientations., https://www.math.ias.edu/ lurie/papers/Elliptic-II.pdf 2018, unpublished.

[Nik16] Thomas Nikolaus, Stable $\infty$-Operads and the multiplicative Yoneda lemma, 2016, arXiv:1608.02901.

[Pér20a] Maximilien Péroux, Coalgebras in the Dwyer-Kan localization of a model category, arXiv e-prints (2020), arXiv:2006.09407.

[Pér20b] Highly structured coalgebras and comodules, https://indigo.uic.edu/articles/thesis/Highly_Structured_Coalgebras_and_Comodules/13475667/1. Aug 2020, PhD Thesis.

[PS19] Maximilien Péroux and Brooke Shipley, Coalgebras in symmetric monoidal categories of spectra, Homology Homotopy Appl. 21 (2019), no. 1, 1-18. MR 3852287

[Swe69] Moss E. Sweedler, Hopf algebras, Mathematics Lecture Note Series, W. A. Benjamin, Inc., New York, 1969. MR 0252485

[Vas19] Christina Vasilakopoulou, Enriched duality in double categories: V-categories and Vcocategories, J. Pure Appl. Algebra 223 (2019), no. 7, 2889-2947. MR 3912953 
Department of Mathematics, University of Pennsylvania, 209 South 33rd Street, Philadelphia, PA, 19104-6395, USA

Email address: mperoux@sas.upenn.edu 